\title{
La coordination entre Bailleurs de Fonds et Maître d'Ouvrage: Le Comité Consultatif de l'Organisation pour la Mise en Valeur du fleuve Sénégal (O.M.V.S.)1
}

\author{
Par Hans-Werner Wabnitz2
}

L'Organisation pour la Mise en Valeur du fleuve Sénégal et ses bailleurs de fonds vivent à l'heure actuelle un moment crucial: la première phase du programme, la construction des barrages, s'achève. L'ère de l'après-barrage a commencé. Il s'agit d'utiliser les infrastructures créées - par les populations riveraines, avec 'aide des administrations locales.

Pour atteindre les résultats escomptés, il faudra encore beaucoup d'efforts de toutes les parties engagées, et d'investissements.

La réussite de la première phase a été rendue possible grâce à la coopération étroite entre le Maître d'Ouvrage et les bailleurs dans un cadre institutionnel appelé "Comité Consultatif" qui garantissait un suivi du projet par les bailleurs, une assistance technique au Maître d'Ouvrage, das un esprit de pragmatisme - une sorte de "task force".

Je suis convaincu que la réussite de l'après-barrage et de la construction du deuxième volet d'ouvrages d'infrastructures dépendra, en large mesure, de la continuité du travail du Comité Consultatif - une continuité qui n'est pas évidente à l'heure actuelle.Pour mieux situer cette coopération entre le Maître d'Ouvrage et ses bailleurs je donnerai, d'abord un bref aperçu

du programme de développement du fleuve Sénégal

des organismes engagés dans ce projet

de la structure de l'Organisation Administrative de l'OMVS

pour ensuite montrer le travail accompli par le ComitéConsultatif jusqu'alors.

\section{Le Programme de Développement de la Vallée du fleuve Sénégal}

Ce programme est "le plus ambitieux d'un développement régional intégré dans l'Afrique des demiers 20 ans" (Marchés Tropicaux du 20 mars 1987 p. 677). La Vallée du fleuve

1 Version retouchée d'un rapport présenté dans le cadre d'un séminaire à l'Université de Bangui du 25 au 27 Mai 1988. Les exposés sont rassemblés dans un recueil édité par l'Université de Bangui (été 1988).

2 Les points de vue exprimés dans la présente note n'engagent que son auteur et ne sauraient être considérés comme l'expression d'une position officielle ou officieuse ni du Haut-Commisariat de l'OMVX, ni des organismes allemands de la Coopération (BMZ; KfW; GTZ). 
Sénégal sépare la Mauritanie au Nord du Sénégal au Sud. La Vallée couvre la région du Sahel. Environ 1,5 million d'habitants y vivent.

Le fleuve prend ses sources dans le Fouta Djallon, en Guinée, traverse le Mali direction $\mathrm{N} / \mathrm{E}$ sur un tiers de sa longueur et toume vers l'Est à la frontière du Sénégal. Il rejoint l'Atlantique à Saint-Louis.

Le régime du fleuve est caractérisé par son irrégularité, il change beaucoup d'une année à l'autre, et durant l'année même - une forte crue après l'hivemage (juillet-novembre) inonde de larges terrains - jusqu'à $50 \mathrm{~km}$ de largeur les années de bonne pluviométrie. Durant la période sèche, par contre, le feluve cesse de couler et l'eau salée de la mer remonte jusqu'à Dagana, 200 km en amont de l'embouchure; la vague des marées se fait sentir jusqu'à 450 $\mathrm{km}$, la moitié de la distance Saint-Louis - Bakel.

Des plans visant à utiliser au maximum les eaux du fleuve et à éviter "une perte" en saison de crue, existent depuis la fin du 19ème siècle. La concrétisation commença quand les Etats riverains Mali, Mauritanie et Sénégal décidèrent en 1972, par décret, que le fleuve Sénégal serait un "Fleuve Intemational" avec comme conséquence la nécessité d'approbation unanime de tout changement de son régime et de toute utilisation d'eau. Simultanément une convention portant création de l'OMVS fut signée.

Cette Organisation, dont les membres sont les trois Etats riverains est chargée

de 'application de la Convention du Statut du fleuve,

de la Coordination et de la planification des Etudes et des travaux dans le cadre du programme.

Le but de ce programme est

de permettre aux populations de la Vallée de rester sur leur terroir en assurant leur autosuffisance alimentaire,

d'éviter une croissance du déficit alimentaire de la Mairtanie et du Sénégal,

de créer des conditions favorables pour l'établissement du commerce, de l'artisanat et de l'industrie dans la région.

Le programme prévoit l'aménagement de plus de 300.000 ha de périmètres irrigués pour le riz (ou toute autre spéculation alimentaire) en double culture.

Ce rideau vert est censé arrêter l'avancement du désert tout le long du fleuve sur une distance d'environ $600 \mathrm{~km}$ et rééquilibrer ainsi un environnement dégradé.

Les ouvrages nécessaires pour atteindre ce but sont

le barrage anti-sel de Diama, à environ 50 km de l'embouchure, en amont de SaintLouis, avec une écluse pour la navigation.

Ce barrage empêche la remontée de la langue salée et permet le remplissage des dépressions naturelles du Delta, notamment le Lac de Guiers qui alimente Dakar en eau potable.

Le barrage fut mis en service après l'hivernage de 85 avec 1 an d'avance sur le programme. 
Ensuite:

le barrage multifonctionnel de Manantali sur l'affluent Bafing, au Mali, réceptionné fin mars 1988 - dans le planning et dans la prévision du budget.

Le bassin retiendra environ $11 \mathrm{mrd} \mathrm{\textrm {m } ^ { 3 }}$ d'eau, soit l'équivalent d'un lac de $500 \mathrm{~km}^{2}$ - comparable au lac de Constance.

La fonction de ce barrage est d'éviter des crues dévastatrices, de garantir l'eau perenne pour l'agriculture et de rendre fonctionnel deux autres ouvrages, qui n'ont pas encore vu le jour et dont le financement n'est pas encore assuré:

la centrale hydroélectrique du barrage de Manantali d'une capacité de $800 \mathrm{GWH}$ (5 turbines à $20 \mathrm{MW}$ ) et

un chenal navigable d'un port de haute mer à Saint-Louis jusqu'à un port fluvial à Kayes, au Mali, soit environ 900 km, avec 11 ports intermédiaires.

Ces ouvrages ont été déclarés propriété commune des Etats-membres par une Convention signée en 1978.

Le Haut-Commisariat agit comme Maître d'Ouvrages de ces constructions - à l'exception des périmètres irrigués qui sont aménagés par les Sociétés Nationales de Développement Agricole et par les Communautés Rurales.

Ces investissements coûtent cher:

les deux barrages représentent environ 205 mrd de FCFA (35 mrd pour Diama, et 170 mrd pur Manantali),

l'électricité demandera environ $100 \mathrm{mrd}$ de FCFA, dont $2 / 3$ pour les lignes de transmission à Dakar,

la voie navigable dans sa première phase coûtera environ 100 mrd de FCFA.

L'aménagement du potentiel des 375.000 ha de périmètres irrigués demandera l'investissement du double de cette somme. Il existe actuellement environ 60.000 ha, coûtant, en moyenne, 5 mio de FCFA l'hectare.

\section{Organismes engagés dans cette Entreprise}

1) D'abord, les trois Etats Riverains: Mali, Mauritanie, Sénégal avec leurs ministères concernés,

2) l'OMVS, dont le siège est à Dakar.

En plus, sur le plan national:

3) des groupes de travail interministériels appelés Cellules de Développement de la Vallée qui se concertent dans le Comité Régional de Planification sous l'égide de l'OMVS,

4) des Sociétés Nationales de Développement Agricole,

5) les Bailleurs de Fonds. 
Parmi ce demier groupe les plus importants se retrouvent au sein de l'OMVS:

les Fonds Arabes - à l'exception de la BADEA - qui financent plus des $2 / 3$ des barrages,

les bailleurs européens - à l'exception de la Banque Européenne d'Investissement et des pays nordiques,

le groupe de la Banque Africaine de Développement,

les Etats-Unis, le Canada et le PNUD.

La Banque Mondiale ne fait pas partie jusqu'alors des bailleurs de l'OMVS, mais elle intervient directement dans l'agriculture de la Vallée, tout comme de nombreuses autres Bailleurs de Fonds et des dizaines d'ONG.

Le programme de développement de la Vallée n'est pas un "Grand Projet" - stigmatisé mais le cadre donné pour mille interventions de tailles moyennes - qui doivent, pour réussir, être planifiées et coordonnées - en principe par l'OMVS.

\section{Organisation de l'OMVS}

1. L'Organe suprême de l'Organisation est la Conférence des Chefs d'Etats et de gouvernement des Etats-membres, qui se réunit tous les deux ans. La commission arrête la stratégie du développement.

Cette Stratégie est transformée en décisions concrètes par

2. le Conseil des Ministres, se réunissant deux fois par an en session ordinaire et chaque fois qu'il est jugé nécessaire en session extraordinaire. Le Conseil représente l'Organisation.

3. L'Organe exécutif est le Haut Commissariat, présidé par le Haut Commissaire. En cas d'absence il est représenté par le Secrétaire Général.

Ensuite, il y a quatre Directions:

de l'Infrastructure Régionale, dont dépendent les barrages,

de l'Investissement,

de la Coordination et de la Planification et

de la Formation et de la Promotion Humaine -

et toute la structure administrative habituelle.

Il y a, en plus, des organes de conseil:

la Commission Permanente des Eaux, une commission interministérielle qui soumet ses recommandations concernant l'utilisation des eaux du fleuve au Conseil des Ministres,

le Comité Inter-Etats de Recherches et de Développements Agricoles - avec trois centres de recherche,

le Comité Inter-Etats de Développement Industriel, le COMITE CONSULTATIF. 
Le Comité Consultatif réunit des représentants des Etats-membres, du Haut Commissariat de 1 'OMVS, et des bailleurs de fonds. Les Sociétiés Nationales de Développement Agricole siègent comme observateurs. La création de ce comité a institutionalisé l'échange continu entre bailleurs et Maître d'Ouvrage.

Conçu comme forum purement technique:

pour faciliter la mobilisation et l'affectation des fonds,

pour améliorer les procédures financières de décaissement et appuyer

l'administration des conventions de financement,

- ce qui n'est déjà pas mince affaire - le Comité s'est développé en un centre de pouvoir qui contrebalance le Conseil des Ministres. En effet, le Haut Commissaire agit souvent en tant qu'arbitre entre le Comité Consultatif et le Conseil des Ministres.

De son habileté d'accommoder les décisions des uns et les conditions des autres, sans blesser les susceptibilités, dépend, dans une large mesure, le bon avancement du programme.

Car, faut-il le souligner - un programme n'est réalisable que si son financement est assuré.

\section{Cadre Institutionnel du Comité Consultatif}

Le Comité Consultatif a été créé le 16 juillet 1975 lorsque la 5ème Conférence des Chefs d'Etat et de Gouvernement à Dakar approuvait son Statut (Résolution No 61 du Conseil des Ministres du 14 juillet 1975). Auparavant la 2ème réunion de l'OMVS avec les 'sources de financement' (les 11 et 12 juillet 1975 à Nouakchott) s'était prononcée en faveur de la constitution d'un tel comité. La 3ème réunion de l'OMVS avec ses 'sources de financement', du 2 au 4 novembre 1977 à Dakar fut la première réunion de Comité Consultatif: elle rédigea sa Réglementation Inteme. Le Conseil des Ministres l'approuva lors de sa 8ème session ordinaire le 5 juin 1978 à Nouakchott (Résolution NO 91).

Le Statut et la Réglementation interne du Comité sont annexés à la présente note. Ils confirment son caractère consultatif: Le Comité émet des avis et des conseils, mais ne décide pas - néanmoins, l'importance de ses membres garantit que ses recommandations ne soient pas négligées. Bien que le Statut et la Réglementation prévoient des 'avis du Comité' impliquant l'unanimité entre bailleurs et OMVS - ces procès verbaux retiennent les opinions des uns et des autres; le plus souvent ils expriment la position d'un ou plusieurs bailleurs, avec en plus le commentaire du Haut-Commissariat. Ces procès-verbaux, établis depuis quelques années d'un commun accord entre Haut-Commissariat et bailleurs, sont soumis au Conseil des Ministres qui succède à ses assises.

Le Comité se réunit une fois par an, en novembre, à Dakar; et chaque fois qu'il est jugé nécessaire, comme, par example, fin mai 1988 pour analyser le volet "énergie".

Son ordre de jour, proposé par le Haut Commissaire, qui préside également les séances, doit être approuvé par le forum. Il comprend l'avancement des différents volets du 
programme, notamment des deux barrages, des études en cours ou à lancer, l'amélioration de la structure du Haut Commissariat, l'état du financement.

\section{L'importance réelle du Comité Consultatif}

Les barrages terminés, l'ensemble de la Vallée peut être maitrisé. Le destin des populations de la Vallée dépend d'une bonne gérance du plan d'eau à Manantali, et d'une assistance efficace dans l'introduction de la culture irriguée.

De la bonne exécution de la suite du programme, et de l'utilisation optimale de ses ressources rendues exploitables, dépendra, en large partie, la survie financière des Etats-membres, lourdement endettés.

Puisque tous les investissements et leur entretien, sont financés par les bailleurs regroupés au sein du Comité Consultatif de l'OMVS, leurs déboursements futurs, et leurs conseils, sont significatifs pour l'avenir de la région.

Par leur engagement pour le programme de développement de la vallée, dont les barrages ne sont qu'un instrument, les bailleurs sont appelés à suivre l'avancement des différents volets et à commenter leurs résultats, car ils restent responsables de la réussite ou de l'échec de ce programme.

Cette responsabilité 'de facto' est naturellement contestée par les bailleurs, qui ne l' acceptent pas 'de jure' - soucieux de limiter leur rôle à celui de prêteurs d'argent.

\section{La Contribution du Comité Consultatif à l'Exécution des Barrages}

Ce qui caractérise le "forum de travail" qu'est le Comité Consultatif est son caractère informel, la souplesse de ses procédures.

Il n'existe à ce jour entre les bailleurs de fonds ni convention, ni consortium, ni chef de file.

Les prêts réunis pour financer les ouvrages sont des financements parallèles, conditionnés les uns aux autres, mais sans liaison directe entre bailleurs.

Dans les sessions du Comité Consultatif, les bailleurs de fonds sont jusqu'à présent intervenus chacun pour son propre compte et ils se sont controntés directement avec le HautCommissariat ou les autres bailleurs. Il y a eu par le passé certaines formes de concertations entre bailleurs, toutefois ce type de concertation est demeuré limité à l'étude et à la solution de problèmes concrets.

Depuis qu'approche le terme de la première phase du projet, on voit se sonstituer différents "blocs": 
d'une part bailleurs de fonds arabes,

d'autre part bailleurs de fonds européens

avec en outre, s'agissant du volet "énergie", l'apparition de la Banque Mondiale, cette demière se proposant aujourd'hui comme chef de file, encouragée en ce sens par les bailleurs représentant la Communauté Européenne.

Dans des discussions portant sur le volet "énergie" ces derniers ont, pour la première fois, parlé en tant qu'Européens, distinctement du FED.

Pour sa part, le Haut-Commissariat a toujours essayé d'éviter la constitution de tels blocs.

Les divergences qui ont pu se faire jour dans le passé entre Haut-Commissariat et bailleurs, ou entre bailleurs, s'agissant soit du mode d'exécution de l'un ou l'autre des barrages, soit des problèmes de déboursements, ont toujours trouvé une solution car elles étaient directement liées à des problèmes précis.

Le Haut-Commissariat craint que la nouvelle tendance à la formation de blocs, ouvre en fait la voie à la prise en compte de considération à caractère politique, à des prises de position de principe, de nature à perturber le travail commun et objectif des experts.

$\mathrm{Car}$, il existe des conflits entre les pays membres, cela les bailleurs en sont conscients, aussi bien qu'entre les bailleurs eux-mêmes.

L'harmonisation de tous les points de vue se fera plus difficilement si des blocs s'affrontent.

Le caractère informel du travail du Comité Consultatif ne l'a pas empêché de trouver des solutions importantes. Au contraire, ce mode de travail vacilite la résolution des problèmes et évite un durcissement des positions. Il évite le reproche 'd'une ingérence' dans les affaires intemes du Maître d'Ouvrages et permet ainsi au Conseil des Ministres d'accepter les propositions des bailleurs. D'un côte il permet aux bailleurs de participer à cet échange de vues sans crainte que ses propositions soient retenues comme engagements formels. Il évite également une attribution des responsabilités pour le programme en plein droit - souci important des bailleurs, comme en témoigne la remarque du représentant de la France lors de la deuxième réunion de l'OMVS avec les 'sources de financement':

"Le Comité sera créé pour renforcer par des avis, des conseils, le Haut-Commissariat, mais ne doit en aucun cas se substituer à lui."

C'est la souplesse de ses procédures qui a permis au Comité de s'adapter aux changements intervenus durant l'exécution du programme, et de garder son efficacité de travail.

\section{Exemples du travail du Comité Consultatif:}

1) Barrage de Manantali et uniformisation des cahiers de charges des ouvrages.

Ainsi le barrage de Manantali n'existe que suite à l'intervention de certains bailleurs qui avaient proposé de régler le régime du fleuve "par la source" et non "par l'embouchure". Il y a eu plusieurs sessions ad hoc en 79 entre ces bailleurs et l'OMVS afin de persuader l'Organisation de se concentrer sur Manantali. Mais, à cette date, les études et préparatifs 
du barrage de Diama étaient trop avancés, des intérêts trop engagés côté Etats-membres et certains autres bailleurs pour en arrêter la construction.

A cause de leurs origines historiques différentes, les cahiers de charges des deux barrages étaient basés sur des conceptions différentes:

contrat modèle FIDIC pour Manantali, contrat modèle FED pour Diama.

Ils ont été uniformisés, sur la base du modèle FIDIC, lequel avait la préférence des bailleurs Arabes, suite à un consensus du Comité Consultatif (6ème, 7ème, 9ème Réunion 1979 et 1980). Cette harmonisation a facilité le suivi d'exécution des deux chantiers.

2) Démarrage des travaux, en l'absence d'un financement totalement "bouclé".

Le Comité Consultatif a permis le démarrage des travaux. Les conventions de financement ne prévoient le déboursement qu'après réunion de la totalité du financement du projet. Dans les années 80, l'OMVS avait du mal à trouver ce financement après le retrait de l'Iran et de l'Irak. Lors d'une réunion extraordinaire du Comité Consultatif, les bailleurs ont approuvé la signature du marché de Manantali, bien que $85 \%$ seulement du financement aient été alors réunis.

L'établissement du plan financier regroupant les 15 bailleurs, intervenant dans des lots divers des ouvrages, n'aurait été possible sans l'intervention étroite du Comité. En plus certains crédits étaient liés.

Le Comité a également facilité le respect des conditions relatives à l'acquisition des biens et services, différents pour chaque bailleur.

3) Décaissement des avances malgré la clause 'pari passu'.

Le décaissement des avances de démarrage aux entreprises s'est trouvé facilité par l'échange de vue dans le Comité Consultatif.

Tout d'abord, pour chacun des deux cantiers, le versement des avances s'est trouvé retardé du fait des difficultés rencontrées dans la mobilisation des fonds. Ces difficultés résidaient essentiellement:

dans la complexité de formalités de mise à disposition des prêts, dans l'organisation parfois peu efficace des administrations des Etats-membres, dans le manque de concertation entre bailleurs et Etats d'une part, et Haut-Commissariat d'autre part, hors des sessions du Comité Consultatif.

Ces délais ont occassionné des intérêts moratoires considérables que les bailleurs ne financent pas et auxquels les états n'ont pas les moyens de faire face.

Or, tous les contrats de financement contiennent la clause dite "pari passu" stipulant une progression égale des déboursements entre bailleurs. On aurait donc dû attendre la réunion des conditions de décaissement du demier bailleur pour effectuer le premier versement. La concertation rendue possible dans le Comité Consultatif a persuadé certains bailleurs de payer des décomptes et des avances pour d'autres bailleurs, dont les condition n'étaient pas encore réunies, et ce jusqu'à $40 \%$ de la totalité du crédit dans un cas - malgré la clause 
"pari passu". Ces bailleurs ont par la suite arrêté les décaissements jusqu'à ce que l'équilibre soit rétabli.

4) Substitution d'un bailleur par un autre en ce qui concerne les déboursements en monnaie locale.

Le paiement en monnaies locales d'un bailleur pour le compte d'un autre dont les statuts interdisaient ces paiements, est un autre exemple de l'utilité de la concertation entre bailleurs, au sein du Comité Consultatif.

5) Non-application de la "cross-default clause".

Durant l'exécution du programme, la concertation des bailleurs a convaicu certains d'entre eux de ne pas apppliquer la "cross-default" clause de leur contrat. Cette clause permet au bailleur de bloquer les déboursements lors d'un retard de paiement par l'emprunteur des commissions, intérêts ou principal exigibles au titre du crédit concerné ou d'un autre crédit de la même source de financement ou d'un co-financier.

Or, les trois états-membres étaient croniquement en retard dans leur remboursement, le plus souvent à propos des crédits bilatéraux ne concernant pas directement l'OMVS.

Les bailleurs arabes faisaient de cette affaire une question de principe. Ils réclamaient rigueur dans la programmation du budget, et exigeaient que soient respectés les engagements pris. Néanmoins l'un d'entre eux s'est montré plus souple à la vue des difficultés créées et, répondant à l'appel du Haut-Commissariat, il a accepté de ne pas appliquer cette clause, pourtant inscrite dans ses propres statuts.

6) Augmentation de l'engagement d'un bailleur pour éviter des dommages.

Il y eut cependant certains blocages. En 1985 notamment, l'assurance de l'entrepreneur de Manantali menaçait de suspendre la couverture du marché, au vu du montant des décomptes demeurés impayés et qui, à ce moment, atteignait plus de $20 \%$ du marché. Pour débloquer la situation, l'un des bailleurs a accepté d'avancer, sous forme d'aide bilatérale, une somme considérable à l'un des pays membres pour lui permettre d'honorer ses échéances envers d'autres bailleurs du projet. Ce faisant, il a permis des décaissements dix fois supérieurs. Cette intervention était sans doute en partie dictée par des considérations d'ordre politique. Je reste cependant convaincu que l'échange constant d'informations par le biais du Comité Consultatif, et la connaissance du mode de raisonnement des autres bailleurs, ont beaucoup contribué à cette initiative.

Ce demier exemple illustre deux points d'une particulière importance, l'un d'ordre général: Le financement des projets complexes destinés à créer des revenus devrait être isolé des autres financements. Ces projets devraient pouvoir être considérés comme une entité propre et obéir à des règles spécifiques, si l'on veut éviter que le manque de liquidité de l'emprunteur ne compromette l'exécution du projet destiné précisément à améliorer sa situation. 
L'autre portant sur le "pourquoi" du succès du fonctionnement du Comité Consultatif:

l'échange constant d'informations entre participants au sein du Comité Consultatif, la communication effective entre bailleurs et Maître d'Ourvrages a constitué pour le projet un atout essentiel. Cette concertation constante a assuré la continuité des travaux.

7) Solution des problèmes techniques.

Outre les décisions d'ordre financier précédemment évoquées, des problèmes à caractère technique ont également été discutés en détail avant le lancement des Marches et au cours de l'exécution du programme.

Les bailleurs ont longtemps débattu:

la hauteur de Manantali pour lui permettre d'accomplir ses différentes missions,

la taille de l'écluse à Diama, pour ne citer que les deux exemples les plus controversés.

Les bailleurs étaient engagés dans la formulation des appels d'offres pour les bureaux d'Ingénieurs Conseils aussi bien que pour les travaux. Ils ont participé dans l'analyse des soumissions. Longtemps, l'idée d'un Conseiller Général fut proposée, en plus des bureaux d'Ingénieurs Conseils pour chacun des deux barrages (9ème réunion 1980 et 11 ème réunion 1981), l'idée fut définitivement abandonnée lors de la 12ème réunion, en janvier 1982.

En cours d'exécution du programme les questions concernant

la quantité des bétons,

le changement à deux reprises de la conception du barrage de Diama,

les mesures à prendre pour éviter des grèves sur les deux chantiers,

occupaient une large place dans les discussions. Questions, qui chaque fois impliquaient le coût des ouvrages, ou de nouveaux risques.

Ces questions étaient de nature à créer des tensions entre bailleurs et Maître d'Ouvrage et, partant, menaçaient directement l'avancement des travaux. Les réunions en Comité Consultatif ont évité qu'elles ne débouchent sur de véritables conflicts. Ce témoignage des sujets traités démontre l'implication directe et détaillée des bailleurs dans l'avancement du programme.

Ces exemples permettent de conclure, que l'engagement commun entre bailleurs et Maître d'Ouvrage de mener à bien ces travaux, et la volonté d'un échange permanent d'information les concemant, ont créé un "esprit de famille" qui a beaucoup aidé à résoudre les problèmes et à surmonter les controverses.

Il ne faut pas sous-estimer l'effet d'un tel esprit, qui a pu voir le jour grâce à la continuité des représentants des bailleurs, au sein du Comité Consultatif durant l'exécution des travaux.

Cet engagement commun n'a pas eu cependant comme conséquence des obligations juridiques définies, analogues à celles découlant d'une "société juridiques définies, analogues à celles découlant d'une "société de fait", ou "joint-adventure". Le résultat en a été la même: 
une volonté délibérée de s'abstenir de toute initiative de nature à compromettre le succès du projet, sinon de le promouvoir.

Un exemple récent illustre qu'au moins un bailleur a sous-entendu une telle concordance de fond. Il estime que le Haut-Commissariat et les autres bailleurs participant au programme, sont obligés de demander l'approbation de chacun des bailleurs pour la construction d'autres ouvrages dans la vallée - même si le financement est déjà assuré.

Dans ce cas précis il s'agit de la Digue Rive Droite, côté mauritanien, partant de Diama jusqu'à Rosso $(80 \mathrm{~km})$. L'OMVS considère cette digue comme faisant partie du "projet Diama". Certains bailleurs par contre considèrent que cette digue constitue un ouvrage à part.

Ce bailleur en question a donc exigé que des études approfondies soient faites concernant le risque de salinité du delta mauritanien créé par la digue. Etudes que l'OMVS a fait exécuter sans inclure les termes de références proposés par ce bailleur.

Deux autres bailleurs ont entre temps accordé le financement nécessaire, utilisant les reliquats des crédits di barrage de Diama. Les travaux devaient commencer en Novembre 1988.

Le bailleur concerné estime que le Haut-Commissariat et les deux financiers n'ont pas respecté leur obligation de lui demander son approbation dans ce cas précis. Il menace d'arrêter tout nouvel engagement au profit de la Mauritanie, bénéficiaire de la future digue.

Cette réaction, bien que négative dans ses conséquences, démontre une attitude conforme aux règles d'une "société de fait", même si de telles obligations n'ont jamais été formellement reconnues par aucun participant au Comité Consultatif.

Considérant, au delà de l'exécution des barrages, le programme d'ensemble du développement de la vallée, il apparaît que l'entente entre les bailleurs est moins apparente.

Les bailleurs ne se sentent pas autant engagés par les autres volets du programme. Ils se considèrent libres de financer des projets opposés à celui-ci:

notamment la route Tambacounda / Bamako, en concurrence directe avec la voie navigable,

ou des centrales thermiques, en concurrence directe avec la centrale hydroélectrique de Manantali,

et ceci, bien évidemment, en accord avec l'Etat-membre bénéficiaire.

Le deuxième exemple est particulièrement délicat. L'énergie de Manantali est la seule ressource capable de rembourser en partie les coûts des ouvrages. Aussi, $48 \%$ des coûts du barrage de Manantali ont été investis en vue d'une telle centrale. Une centrale thermique, placée au centre du marché visé, risque d'empêcher la rentabilité d'une centrale hydroélectrique, décourageant ainsi d'autres bailleurs à la financer. Est-ce que les deux Etats-membres étrangers à la décision de construire une centrale thermique pourront, un jour, refuser le remboursement, parce que les bailleurs les ont activement empêchés- de rassembler les moyens nécessaires?

La tendance de se retirer de la poursuite du programme de développement, pourtant communément approuvé, s'avère donc dangereuse pour l'avancement de l'ensemble. Elle 
devrait être freinée par une planification et coordination plus rigoureuse des investissements dans la région par l'OMVS.

Mais les partenaires s'y opposent:

les Etats craignent une perte de pouvoir de décision et par là de souveraineté;

les directions des bailleurs craignent une perte de liberté d'agir et de choisir entre différents projets. Ils ne veulent surtout pas accepter une éventuelle obligation de continuer à financer les volets qui restent au programme. Une conséquence qui est pourtant tacitement impliquée dans la participation au financement des barrages.

Les membres du Conseil des Ministres agissent souvent en tant que défenseurs des intérêts particuliers de leurs Etats, et non comme avocats d'un nouvel avenir sub-régional. L'OMVS n'est donc pas bien en mesure de palier à cette tendance.

Cette perspective moins optimiste pour la poursuite du programme de l'OMVS souligne: primo, l'effort que le Haut-Commissariat a mis en oeuvre pour concentrer des ressources humaines et financières afin de mener à bien l'exécution des barrages; segundo, le besoin de redoubler cet effort pour continuer le programme tel qu'il a été conçu, la nécessité de poursuivre avec plus de rigueur encore la sensibilisation des partenaires: les Etats et les bailleurs.

Le cadre privilégié de concertation entre l'OMVS et ses bailleurs qu'est le Comité Consultatif est donc à maintenir et à fortifier.

Dans ce même esprit, encouragé par le "rôle fort constructif de coordination entre les bailleurs et l'OMVS d'un côté, et entre les bailleurs eux mêmes de l'autre côté (du Comité Consultatif)" le groupe des bailleurs arabes ${ }^{3}$ propose l'établissement d'un 'Comité Technique' à l'image du Comité Consultatif pour la poursuite du programme. Car

"il est du devoir des Etats-membres et des bailleurs de fonds (ayant participé au programme) de trouver des moyens pour accélerer le développement des projets, profitant de la mise en eau de Manantali, et de trouver des utilisations toujours plus efficaces de l'eau retenue dans le barrage, pour améliorer les perspectives du programme."

La continuité du travail du Comité Consultatif, dans le cadre élargi du programme de développement de la Vallée du fleuve Sénégal, pourrait contribuer, d'une manière importante, à canaliser les ressources, limitées aux projets prioritaires, et à éviter une multiplication d'études et de projets, ou pire encore, l'exécution de projets contradictoires.

3 Note: "Experience in a regional project: the case of the Senegal river basin development (OMVS)" prepared by the Arab national and regional development institutions, Islamic Development Bank and OPEC Fund, for the DAC / ARAB Group workshop on the coordination experience in Senegal, Dakar, 9-10 feb. 1987. 
established procedures for sale of land and its use as a collateral for a loan had already emerged in the colonial days.

Today, conditions for the production of cocoa are such that it can possibly be a motor for the country's economic development.

The paper discusses the attempts after the coup d'état of 1979 to redistribute the land. Again, the parallels to the situation in Latin America are striking. Land is increasingly organized into large estates owned by the administrative class, whereas those who work it are left basically landless. The author argue that only a land reform -- among other necessary conditions -- that addresses this imbalance can provide the incentive to put cocoa once more in its place as an engine of growth.

The Coordination between Silent Partner and Owner:

A Consultative Committee for the Development of of the Senegal River

By Hans-Werner Wabnitz

The author presents a revised version of a lecture held at the international seminar on the role of law regarding to financial development and investments in Africa, 25.-27.5.1988 at Bangui University. The lectures are condensed in a volume edited by the University of Bangui, summer 1988.

\section{ECOWAS - Economic Community of West African States}

By S.B. Ajulo

ECOWAS has become an exemplarly economic community as for the first time one has succeeded in creating a community of African States with different colonial background. Problems will arise still, but if the community stands the test this will be a chance to the future and a contribution by West Africa to international commercial law in general and international law in particular. Should this trial for cooperation fail, another arrangement would be made. As the social structure of West African States is intermingled the current economic dependency on each other will grow thus making the political borders a potential menace to peace. 